\title{
AN ADAPTIVE SHIP DETECTION ALGORITHM FOR HRWS SAR IMAGES UNDER COMPLEX BACKGROUND: APPLICATION TO SENTINEL1A DATA
}

\author{
Guangjun $\mathrm{He}^{1}$, Zhenghuan Xia ${ }^{1}$, Hao $\mathrm{Chen}^{1}, \mathrm{Ke} \mathrm{Li}^{1}$, Zhilong Zhao ${ }^{1}$, Yuhua Guo ${ }^{1}$, Pengming Feng ${ }^{1,} *$ \\ ${ }^{1}$ State Key Laboratory of Space-Ground Integrated Information Technology, Beijing Institute of Satellite Information \\ Engineering, Beijing 100029, China - hegj@sgiit.cn
}

KEY WORDS: HRWS SAR, Real-time ship detection, Adaptive sliding window, GLCM, Sentinel1A

\begin{abstract}
:
Real-time ship detection using synthetic aperture radar (SAR) plays a vital role in disaster emergency and marine security. Especially the high resolution and wide swath (HRWS) SAR images, provides the advantages of high resolution and wide swath synchronously, significantly promotes the wide area ocean surveillance performance. In this study, a novel method is developed for ship target detection by using the HRWS SAR images. Firstly, an adaptive sliding window is developed to propose the suspected ship target areas, based upon the analysis of SAR backscattering intensity images. Then, backscattering intensity and texture features extracted from the training samples of manually selected ship and non-ship slice images, are used to train a support vector machine (SVM) to classify the proposed ship slice images. The approach is verified by using the Sentinl1A data working in interferometric wide swath mode. The results demonstrate the improvement performance of the proposed method over the constant false alarm rate (CFAR) method, where the classification accuracy improved from $88.5 \%$ to $96.4 \%$ and the false alarm rate mitigated from $11.5 \%$ to $3.6 \%$ compared with CFAR respectively.
\end{abstract}

\section{INSTRUCTIONS}

Ship detection is of great significance for maritime surveillance, fishery activity management and illegally operating ships monitoring (Ouchi, 2013). During the past years, Synthetic Aperture Radar (SAR) has become a crucial method for ship detection (Moreira, 2000). Compared to the optical, infrared and hyperspectral sensors, SAR is leading in imaging capability in anytime and any weather conditions. In particular, the features of metal material ships are more obvious for detection with ocean background. This mirrors out the specified the advantages of employing SAR systems in ship surveillance (Roarty, 2011). As the development of the High Resolution Wide Swath (HRWS) on board SAR systems in recent years, the conflicts between the high resolution and wide swath have been solved successfully, thus marine target surveillance by HRWS SAR system are becoming possible (Margarit, 2009; Brusch, 2011).

Currently, algorithms based on Constant False Alarm Rate (CFAR) are widely used for ship detection (Goldman, 1988; Hwang, 2010; An, 2014). Among them, the most popular methods are two parameter CFAR, K-distribution CFAR and KSW double threshold method (Gao, 2017; Ji, 2015). Two Parameter CFAR algorithm employs the Gaussian distribution to estimate the background clusters, and three sliding windows are employed to go through the images. Therefore, the image is segmented to scattering regions according to the local threshold. However, ship detection rate is reduced due to the sea clutter distribution, which is hard to be illustrated by Gaussian distribution properly under circumstances of terrible ocean environment. K-distribution CFAR utilizes k-distribution instead of the Gaussian distribution to characterize the sea clutters, to make good illustration on the tailing phenomenon of the sea clutters during terrible ocean environment by adopting its shape parameter. However, the parameters of the k-distribution are uncertain and difficult to be estimated accurately. KSW double threshold method uses the idea of information entropy into the image segmentation. The methodology can be described as to find the maximum value of the sum of the information entropies after image classification. In this case, strong Bragg resonance will occur under the terrible ocean environment, especially when ocean surface is accompanied by strong wind. Therefore, KSW double threshold method is easily effected by false alarm, the noise with higher reflection can be recognized as the target in majority of the cases (Gandhi,1988; Frery, 1997; Smith, 2000; Marimo, 2014). Furthermore, a popular ship detection algorithm usually consists of three steps: land masking, prescreening and discrimination (Enayati, 2015). The land masking stage is always based on transcendental knowledge, which severely restricted the real-time processing on the satellite.

In terms of the problems mentioned above, this paper presents an adaptive algorithm for ship detection in the ocean by using the HRWS SAR images. Firstly, the SAR image slices of the nonwater area (including ships, islands, island-reefs, drilling platform, seawater farms, etc.) are obtained by water-land segmentation. Then, a classifier trained by the GLCM texture features of the manually selected ship and non-ship slice images is employed to classify the slices to ships and non-ships. Accordingly, the ships can be detected under complicated ocean conditions.

\section{METHODOLOGY}

A flowchart summarizing the method proposed in this study is presented in Fig. 1, including the following steps: water body extraction, ship areas proposal, GLCM texture feature extraction and SVM classification.

\footnotetext{
* Pengming Feng, Email:p.feng.cn@outlook.com
} 


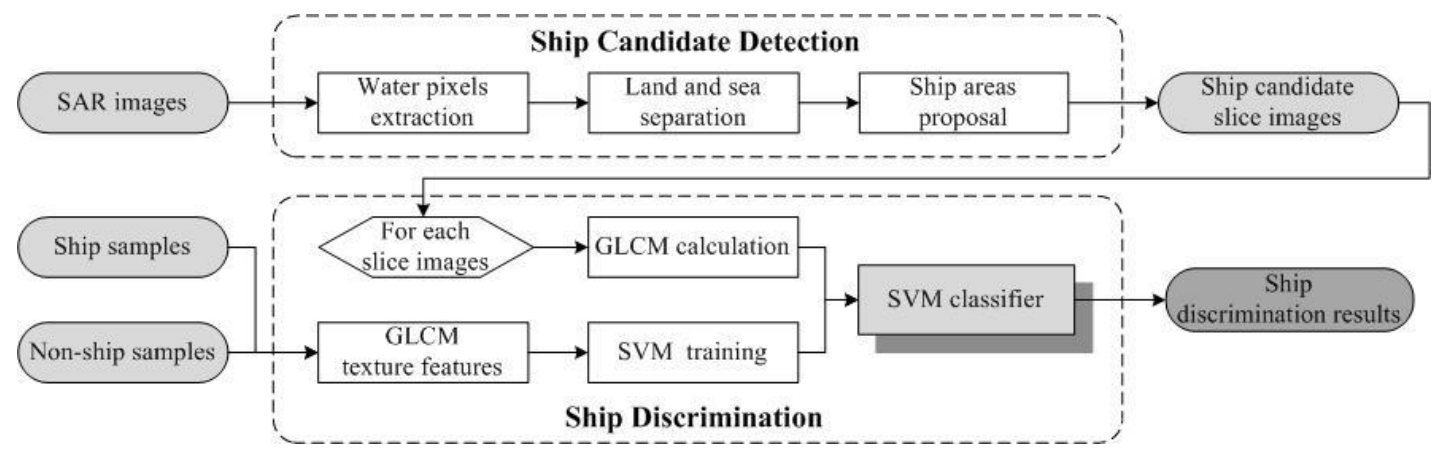

Fig. 1 Schematic flowchart illustrating the ship discrimination method adopted in the present study.
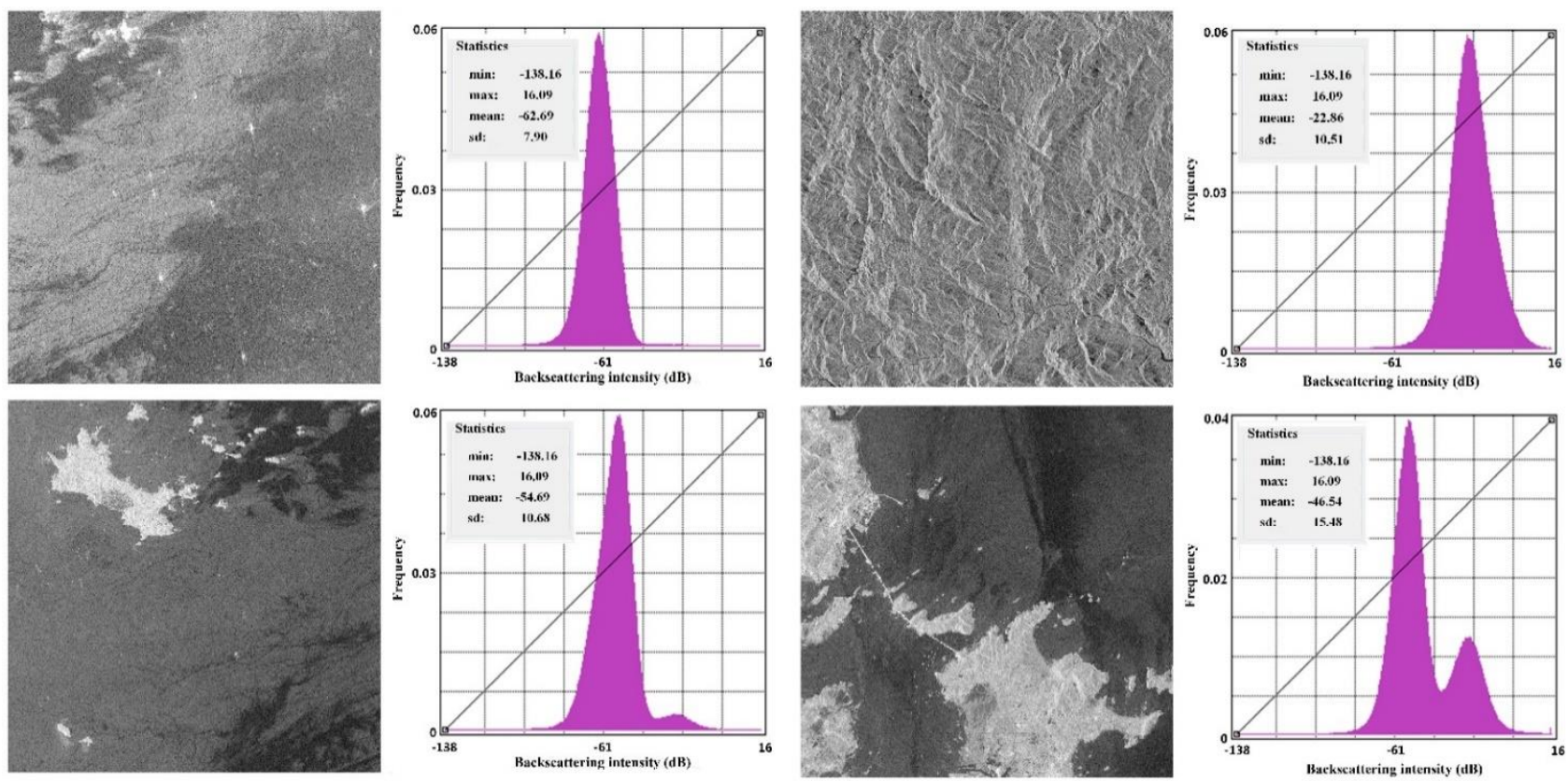

Fig. 2 Backscattering intensity value comparisons for land and sea areas.

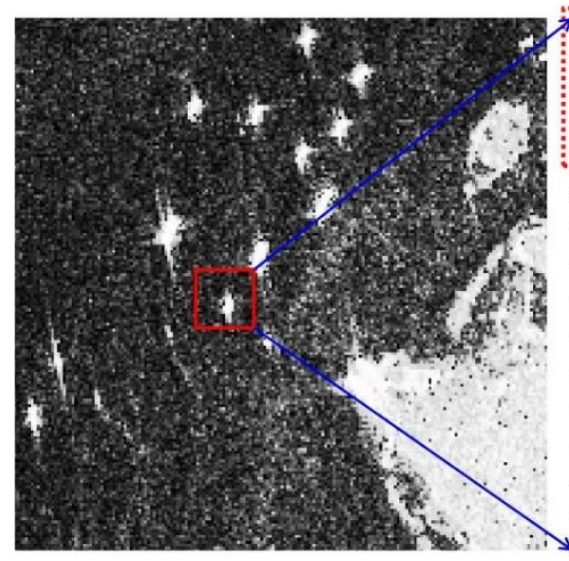

(a)

\begin{tabular}{|c|c|c|c|c|c|c|c|c|c|c|c|c|}
\hline 51 & -50 & -57 & & 57 & 55 & \begin{tabular}{l|l|l}
56 & -50 \\
\end{tabular} & \begin{tabular}{|l|} 
\\
\end{tabular} & & -55 & & & \begin{tabular}{r|l|l}
54 & -51 \\
\end{tabular} \\
\hline-58 & -61 & & & & 61 & 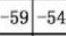 & -47 & & -58 & -55 & 62 & \begin{tabular}{l|l}
-60 & -66 \\
\end{tabular} \\
\hline 57 & -48 & & 6 & 5 & 68 & \begin{tabular}{l|l}
-51 & -45
\end{tabular} & -54 & -51 & -71 & -55 & -54 & \begin{tabular}{l|l}
-56 & -53
\end{tabular} \\
\hline 60 & -53 & 55 & -58 & 76 & $-57]$ & \begin{tabular}{l|l}
-51 & -42
\end{tabular} & 51 & 54 & -57 & 68 & $-58 \mid=$ & \begin{tabular}{l|l}
-52 & -54
\end{tabular} \\
\hline 58 & 52 & 5i & 65 & -56 & -55 & \begin{tabular}{l|l}
-49 & -42
\end{tabular} & -35 & -80 & -48 & -4 & $-48-=$ & \begin{tabular}{l|l}
-55 & -63
\end{tabular} \\
\hline 66 & & 46 & -47 & -57 & -54 & \begin{tabular}{l|l}
-37 & -28
\end{tabular} & -16 & -57 & -43 & -5 & $-44-$ & \begin{tabular}{l|l}
-63 & -56
\end{tabular} \\
\hline 60 & & & -5 & -47 & -38 & \begin{tabular}{l|l}
-46 & -12
\end{tabular} & -5 & 27 - & -56 & -46 & $-45 \mid-$ & \begin{tabular}{l|l}
-48 & -49
\end{tabular} \\
\hline-56 & & 56 & -62 & -49 & -42 & \begin{tabular}{l|l}
-69 & -1
\end{tabular} & 5 & -26 & -48 & -5 & $-53 \mid-$ & \begin{tabular}{l|l}
-53 & -56
\end{tabular} \\
\hline 54 & -53 & 45 & 5 & -48 & -68 & \begin{tabular}{l|l}
-16 & 12
\end{tabular} & -9 & -25 & $|-55|$ & -44 & -52 & \begin{tabular}{l|l}
54 & -60
\end{tabular} \\
\hline-6 & -54 & -55 & -52 & -47 & -44 & \begin{tabular}{l|l}
-15 & 13
\end{tabular} & -7 & -30 & $\mid-50$ & -53 & -50 - & \begin{tabular}{l|l}
-67 & -62
\end{tabular} \\
\hline 5 & -53 & -69 & 61 & -51 & -45 & \begin{tabular}{l|l}
-40 & -11
\end{tabular} & \begin{tabular}{|l|}
-17 \\
\end{tabular} & \begin{tabular}{|c|}
-52 \\
\end{tabular} & $\mid-52$ & -53 & -52 & \\
\hline-5 & -54 & -60 & & -50 & -55 & \begin{tabular}{l|l}
-71 & -36
\end{tabular} & $\mid-33$ & & $\mid-51$ & -48 & -57 & \begin{tabular}{c|c|c|}
-53 & -61
\end{tabular} \\
\hline 5 & -56 & & & -50 & -55 & \begin{tabular}{l|l|l}
-69 & -1
\end{tabular} & $\mid-26$ & -52 & $\mid-48$ & -53 & & \\
\hline & -5 & & & 61 & 56 & \begin{tabular}{l|l}
-70 & -44
\end{tabular} & -37 & & $\mid-54$ & & & \\
\hline & & & & & & \begin{tabular}{c|c|c|}
-69 & -5
\end{tabular} & & & & & & \\
\hline
\end{tabular}

(b)

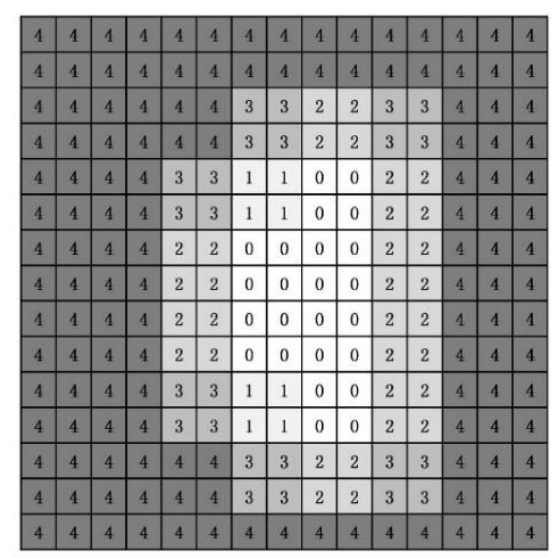

(c)

Fig. 3 Schematic diagram of sliding window for the labeling of water pixels, where (a) presents the backscattering coefficient images, (b) present the backscattering intensity value and the sliding window, (c) presents the number of marks for each pixel.

\subsection{Water Body Extraction}

In this paper, the adaptive sliding window has been applied. The water areas are segmented by labeling each pixels of the image using the sliding window. Moreover, the size of sliding window, the length of sliding steps, the uniformity of the pixels in each window and the hard threshold were determined according to the backscattering intensity histogram of the images in this algorithm. A study on the backscattering coefficients of SAR images has shown that the scattering intensities value of ocean are significantly different from land (island, island-reef) (Migliaccio, 2014). Figure 2 shows an example of the backscattering coefficients of the SAR images for ocean and land area, which both follow the Gaussian distribution. However, the scattering 


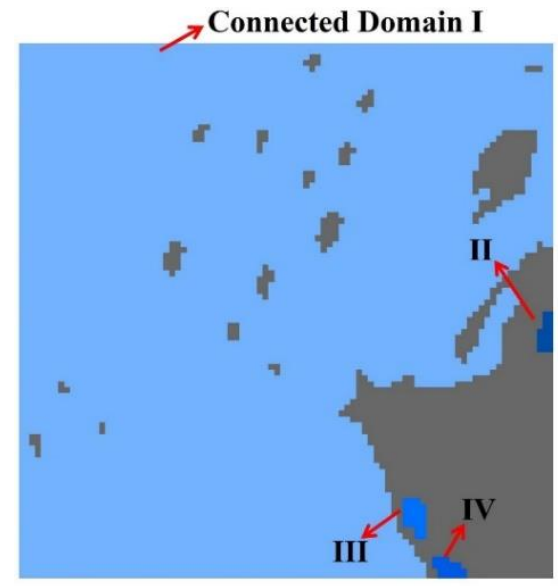

(a)

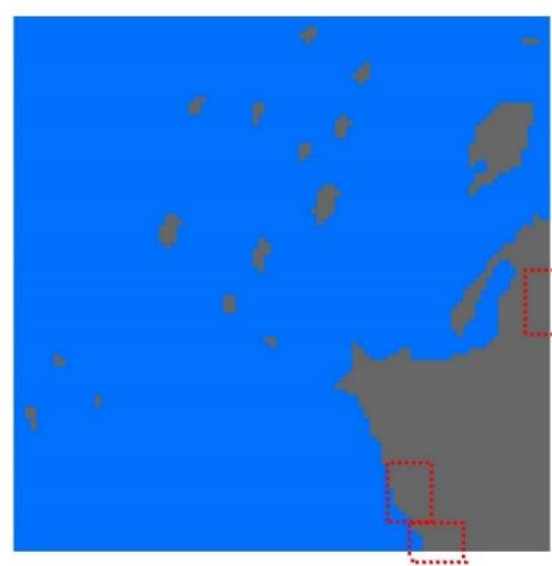

(b)

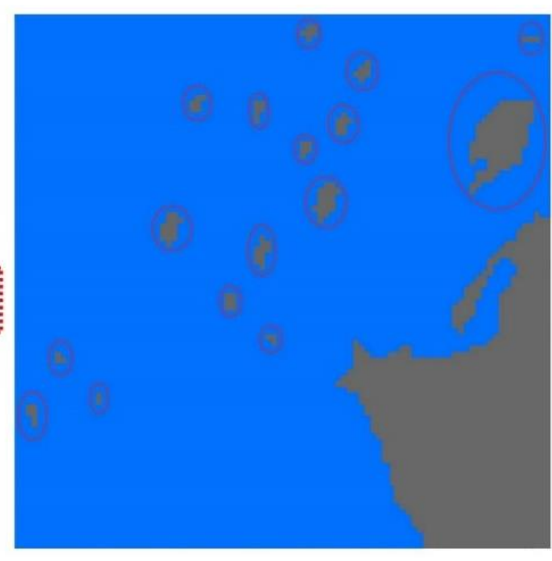

(c)

Fig. 4 Schematic diagram of ship areas proposal based on the water pixels labeling results, where (a) represents the extracted water pixels, (b) represents separation results of land and sea, and (c) represents ship areas proposal result.

intensity of the water and land scene share different average value and standard deviation, which are $-62.69 \mathrm{~dB}$ and 7.9 for water and $-22.86 \mathrm{~dB}$ and 10.51 for land respectively. For the SAR images with water scene or land scene, the average scattering intensities of $95 \%$ of the pixels within the image with the standard deviation error of 2 plus minus. Therefore, the uniformity of the pixels and the hard threshold of the water scattering intensity can be determined by features of the backscattering intensities histogram of the images. The size of the sliding window is related to the size of the ships which is required to be detected. Generally, the smaller sliding steps, the clearer boundary contour, but this will lead to higher computational expenses. Thus, the size of the smallest ship needs to be set in advance and will be used as the length of the sliding steps of the sliding window, and the size of the sliding window should be twice of the sliding steps in order to ensure a $50 \%$ overlap. Figure 3 illustrates a schematic diagram of sliding window for the labelled water pixels.

\subsection{Ship Areas Proposal}

When using the water body extraction algorithm mentioned above to label the full images, the small water areas and inland areas such as mountain shadows with low scattering intensities will be labelled as the water areas. In order to obtain the complete ocean scene, post-processing is required for the labelled water pixels. Compared to land scene, water region in ocean scene has the characteristics such as stronger connectivity, larger area, lower scattering intensity and higher homogeneity. Based upon these characteristics, 8-connected field of the labelled water region is firstly formed, as is shown in Fig. 4a, then the areas of in-land water are removed based upon the size of the connected domain and the number of times marked as water pixel, the result is shown in Fig. 4b. After combining the in-land water areas and the land areas, the remaining regions in the image can be regarded as ocean scene and non-ocean scene, where the non-ocean scene contains targets such as ships, land, island, island-reef, etc. Finally, the areas with a large size are removed based on a hard threshold.

\subsection{GLCM Textural Features Extraction}

Gray-level co-occurrence Matrix (GLCM) represents the spatial dependence of the gray-level and the spatial relationship of gray pixels in a particular textural mode (Anfinsen, 2011), which performs well especially when presenting local texture in small areas. GLCM are normally square matrix, the dimension of which is equal to the gray-level of the image. Given two pixels with grayscale $i$ and $j$, and the distance between the two pixels is d with relative directions, element $G(i, j)$ presents the times of these two pixels appear in the image. Practically, the value of $\theta$ is chosen as $0^{\circ}, 45^{\circ}, 90^{\circ}$ and $135^{\circ}$. Generally, the grayscale of a gray image is 256 . However, the textural features can hardly be presented when the grayscale of GLCM is too high and the size of window is small. Meanwhile, the computational complexity increases exponentially. In this case, the image requires to be zoomed based upon its grayscale before computing GLCM. Empirical value of 16 is selected for the grayscale and 4 kinds of eigenvalues are calculated for GLCM, including angular second moment $(A S M)$ energy, entropy (ENT), inverse difference moment $(I D M)$ and correlation $(C O R)$, which are respectively expressed as (Anfinsen, 2011):

$$
\begin{gathered}
A S M=\sum_{i=1}^{k} \sum_{j=1}^{k}(G(i, j))^{2} \\
I D M=\sum_{i=1}^{k} \sum_{j=1}^{k} \frac{G(i, j)}{1+(i-j)^{2}} \\
\text { ENT }=\sum_{i=1}^{k} \sum_{j=1}^{k} G(i, j) \log (G(i, j)) \\
C O R=\sum_{i=1}^{k} \sum_{j=1}^{k} \frac{(i j) G(i, j)-u_{i} u_{j}}{S_{i} S_{j}}
\end{gathered}
$$

Where $k$ is the grayscale. Mean and standard deviation (SD) of the four eigenvalues were finally selected as the parameters for the texture comparisons between ship and non-ship slice images, as is shown in Fig.5. Jeffreys-Matusita (J-M) (Bruzzone, 1995) distance was applied for the parameter selection combined with the acquired ship and non-ship slice images. The J-M distance of the parameters can be expressed as follows:

$$
\begin{gathered}
J=2\left(1-\mathrm{e}^{-B}\right), \quad 0<J<2 \\
B=\frac{1}{8}\left(m_{1}-m_{2}\right)^{2} \frac{2}{\delta_{1}^{2}+\delta_{2}^{2}}+\frac{1}{2} \ln \left[\frac{\delta_{1}^{2}+\delta_{2}^{2}}{2 \delta_{1} \delta_{2}}\right]
\end{gathered}
$$

Where $J$ is the $\mathrm{J}$-M distance for the parameter, $m_{1}$ and $m_{2}$ are the average values of the parameter corresponding to ship and nonship slice images, $\delta_{1}$ and $\delta_{2}$ are the variance. We calculated the J-M distance for all the 12 parameters, including the maximal 

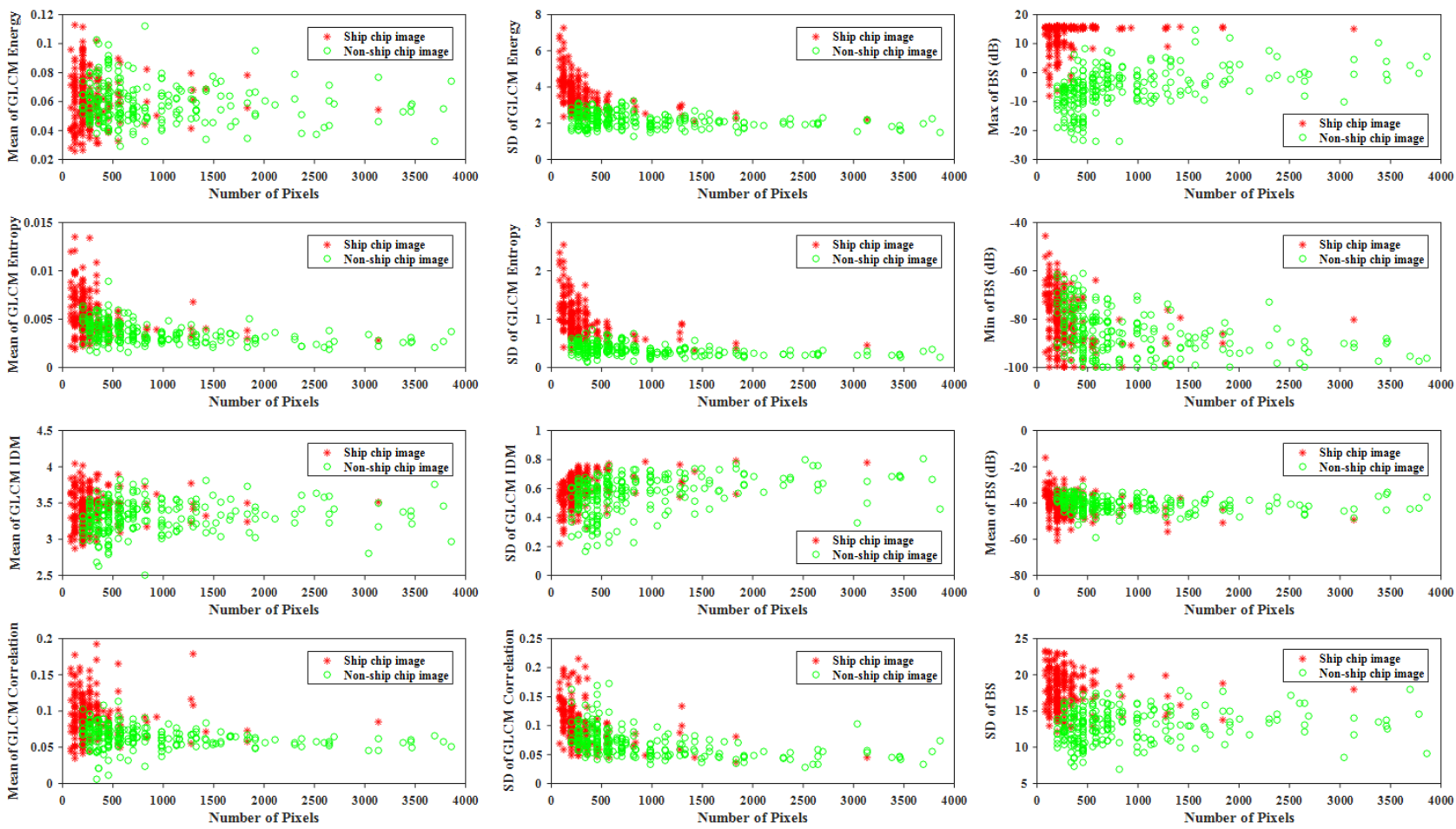

Fig.5 Features analysis for ship and non-ship slice images.

value, minimum value, average value and standard deviation of backscatter intensity of the slice images, the results showed that $\mathrm{J}-\mathrm{M}$ distance for standard deviation of energy and entropy, maximal value of backscatter intensity are above 1.9 , therefore, the three parameters are selected as the optimal combination for the classification of ship and non-ship slice images.

\subsection{Ship discrimination}

The support vector machine (SVM) is a classifier that is widely used owing to its ability to solve nonlinear classification problems with small samples and high dimensions (Camps, 2005). The SVM classifier is typically constructed based on a small number of training instance-label pairs, which are used to search for the support vectors and then to predict unknown data. For a two-class problem, it can be assumed that the training instance-label pairs are $\left\{\left(x_{i}, y_{i}\right), i=1, \ldots, \mathrm{N}\right\}$. Each training instance-labelled pair $\left(x_{i}, y_{i}\right)$ is a vector in the d-dimensional feature space $x_{i}=\left[x_{i, 1}, x_{i, 2}, \ldots, x_{i, d}\right]^{\mathrm{T}}$ with a corresponding label $y_{i}$. The decision function in the kernel space can be expressed as follows:

$$
f(x)=\sum_{S} a_{i} y_{i} K\left(x_{i}, x\right)+b
$$

where $f(x)$ represents the margins. $S=\left\{i: 0<a_{i}<C\right\}$, where $C$ is a penalty parameter. Samples associated with nonzero $a_{i}$ are so-called support vectors. $b$ is a bias term that has low effect on the performance. $K\left(x_{i}, x\right)$ is the kernel function; in the present study, a Gaussian RBF kernel was employed. This kernel took the following form:

$$
K\left(x_{i}, x\right)=\mathrm{e}^{-\gamma\left\|x_{i}-x\right\|^{2}}
$$

where $\gamma$ is a parameter that is inversely proportional to the width of the Gaussian kernel. The two parameters $C$ and $\gamma$ were not known beforehand, but grid-search and cross-validation methods were used to search for the best parameters (Bazi, 2006).
Subsequently, the training instance-label pairs are used to confirm the support vectors.

1500 ship slice image and 1500 non-ship slice images are selected as the patterns. The patterns are divided into 3 subsets with equal size. One subset is used to test the classifier trained by the other two subsets. After the grid search conducting using 3fold cross-validation, the best values of the two parameters are identified. Then, the classifier for ship discrimination is finally built.

\section{EXPERIMENTAL RESULTS}

The proposed approach is verified using a series of C-band Sentinl1A data on interferometric wide swath mode with a swath width of $250 \mathrm{~km}$ and a ground resolution of $5 \mathrm{~m} \times 20 \mathrm{~m}$ (range $\times$ azimuth). This mode images in three sub-swaths using the terrain observation with progressive scans SAR. With this technique, the radar beam scans back and forth three times within a single swath, resulting in a higher quality and homogeneous image throughout the swath. These data were acquired respectively on March 26, 2016, April 26, 2016, June 30, 2016, and July 24, 2016 respectively, around the coastal area of Fujian, China. The images were processed using the SARScape software. Firstly, the metadata files of Sentinl1A data are imported into the software to generate the SLC mosaic images. The SLC images are multilooked four times in range direction so that the spatial resolution are reduced to $20 \mathrm{~m} \times 20 \mathrm{~m}$ (range $\times$ azimuth). Then, geocoding are conducted with the ASTER GEDM V2 product and the backscattering coefficient images are projected onto the DEM coordinate system. Finally, the images are sliced into image blocks of size $1440 \times 1200$.

The sliding window with size of $6 \times 6$ is employed for water extraction and the sliding step is set to three. Uniformity threshold of the pixels in the window is set to be $95 \%$, which means that the numbers of the pixels with the scattering intensity 


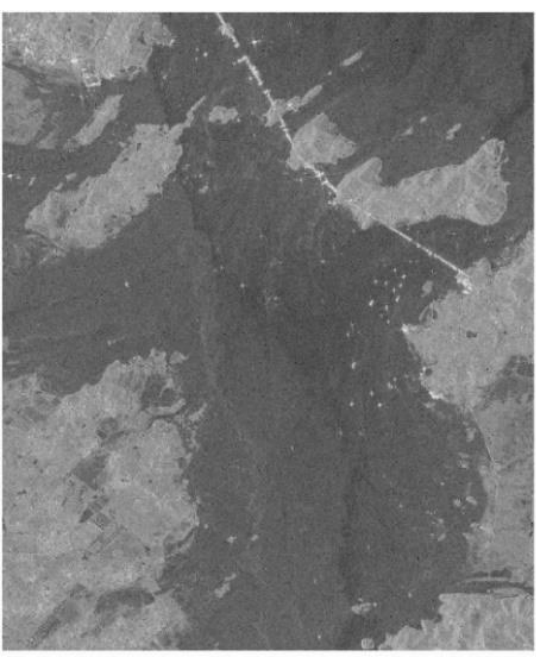

(a)

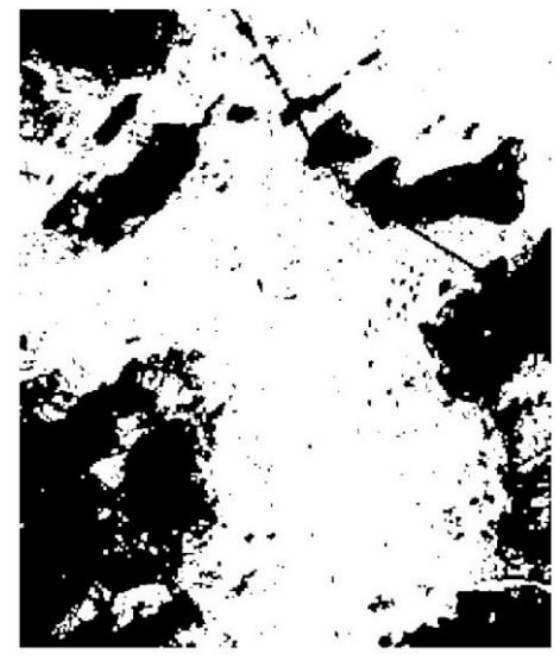

(b)

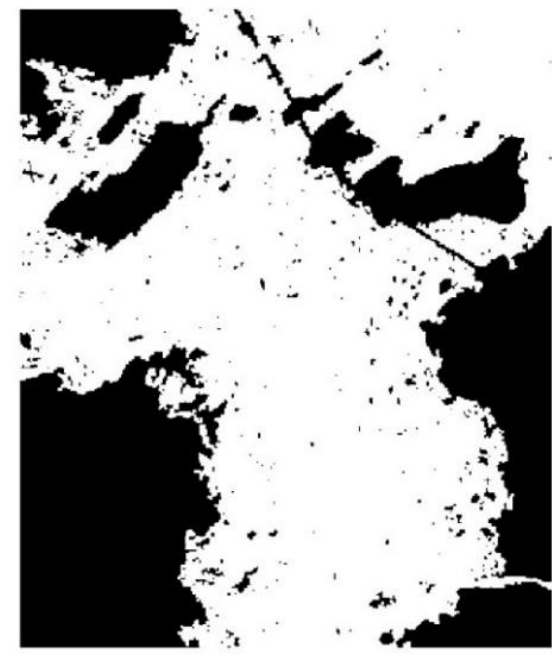

(c)

Fig.6 Land and sea separation. (a)SAR backscattering coefficient image, (b) water pixels extraction results, (c) land and sea separation results.

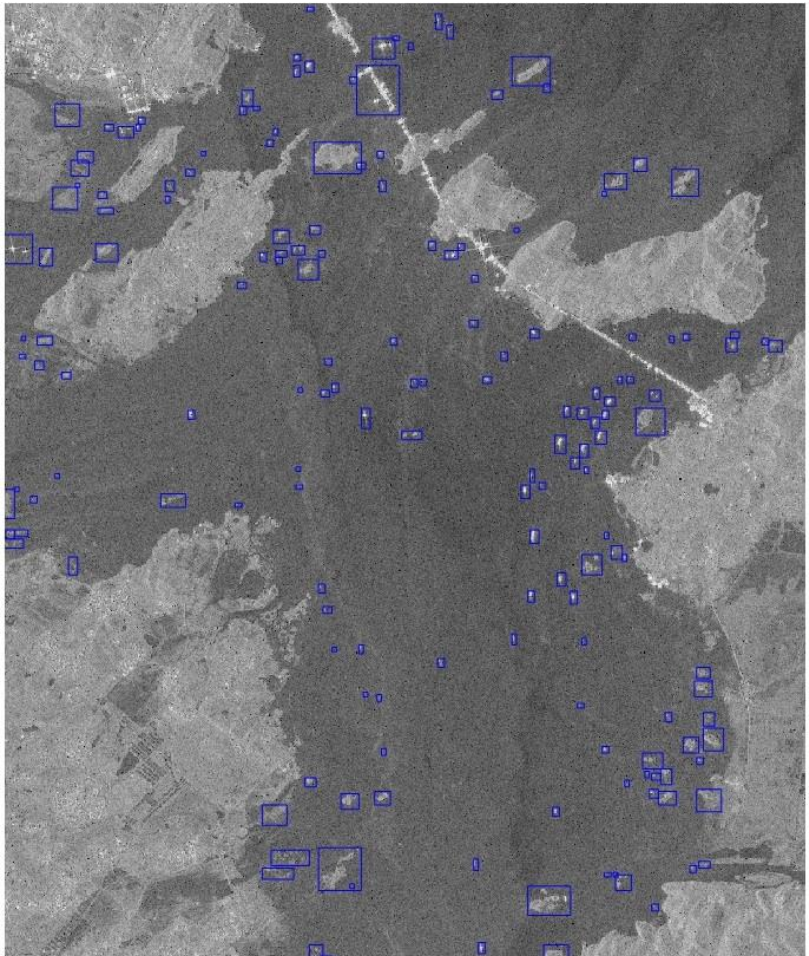

(a)

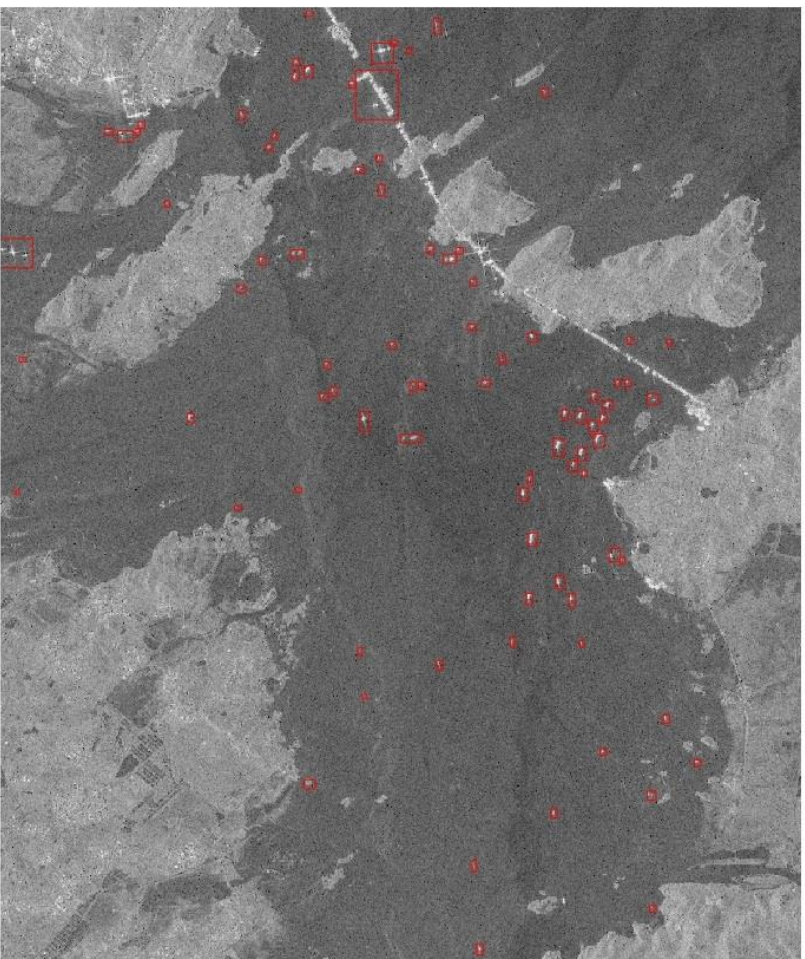

(b)

Fig.7 Experimental results. (a)Ship areas proposal results, (b)ship detection results.

larger than the threshold should be less than one. Connected domain was built by the pixels labelled as water, and pixels in the connected domain should be labeled at least 2 times averagely, to be finally determined as water areas, the results is shown in Fig. $6 \mathrm{~b}$. Then, water connected domain with total pixel number less than 17200 ( $1 \%$ of the sliced image) are merged into the land region, and the remaining regions are assumed to be ocean scene region, as is shown in Fig. 6c. The binary image are generated according to the water extraction results, and the non-water connection region is achieved. The ship proposal areas are selected from non-water connection region with total pixel number less than 4000, as is shown in Fig. 7a. After achieving the ship proposal areas, standard deviation of GLMC energy and entropy are calculated for each area, then the pre-trained SVM classifier is utilized to classify the areas into ship and no-ship, the results is shown as Fig. 7b.

The ship areas proposal and ship discrimination results were validated with google map data, considering the google map labeled results (land areas, islands and reefs, marine farms, etc.) as the "ground truth". The accuracy calculation formula of ship areas proposal and ship detection, respectively, expressed as the division of the number of proposal offshore ships and the total number of offshore ships, and the division of the number of extracted offshore ships and the total number of offshore ships. The total accuracy of ship areas proposal is found to be $99.3 \%$, 
and the accuracy of ship detection is found to be $96.4 \%$ for the selected four images.

The results indicate that the method provides an effective means for ship discrimination by weaken the influences of land area and non-ship targets at sea. Furthermore, the accuracy and false alarm rate are obviously superior to those achieved by the typical constant false alarm rate (CFAR) algorithm (88.5\% and $11.5 \%$, respectively). However, as is shown in Fig. 7, ships anchored at ports or close to the shore are not discriminated by using the proposed method, this is because the ships have being merged into the land area in the section of connected domain constructing. Nevertheless, this method has been shown to be useful for identification of offshore ships.

\section{CONCLUSIONS}

In this study, a novel approach based on water extraction and SVM was presented for ship discrimination by using HRWS SAR data. In particular, an adaptive sliding window was developed to extract water pixels and then to propose the ship areas based on the analysis of SAR backscattering intensity images of different marine scenes, and a SVM classifier is built based on the GLCM texture features to classify the proposed ship slice images into ships and non-ships. This newly developed method achieved the accuracy of $99.3 \%$ for ship areas proposal and accuracy of $96.4 \%$ for ship discrimination, offering significant improvement over the typical constant false alarm rate algorithm. In addition, all parameters of the algorithm were extracted on the basis of the acquired SAR data, and required no geographical based map or empirical parameters. However, ships close to shore were usually merged into the land area in the process of water area extraction. Accordingly, future work should be focus on the discrimination of the ships close to shore.

\section{ACKNOWLEDGEMENTS}

The authors would like to thank the ESA for the free Sentinel1A data, and the anonymous reviewers for their helpful comments and suggestions.

\section{REFERENCES}

K. Ouchi., 2013. "Recent trend and advance of synthetic aperture radar with selected topics," Remote Sensing., vol. 5, pp. 716-807.

A. Moreira, P. Prats-Iraola, M. Younis, G. Krieger, I. Hajnsek, and K. P. Papathanassiou., 2013 "A tutorial on synthetic aperture radar," IEEE Geoscience and Remote SensingMagazine, vol. 1, no.1, pp. 6-43,

G He, X Feng, P Xiao, Z Xia, Z Wang, H Chen H Li, and J Guo, 2017. "Dry and Wet Snow Cover Mapping in Mountain Areas Using SAR and Optical Remote Sensing Data", IEEE Journal of Selected Topics in Applied Earth Observations and Remote Sensing,vol. 10, no. 6, pp. 2575-2588.

H. J. Roarty, E.R. Lemus, E.Handel, S. M. Glenn,D. E. Barrick,and J. Isaacson,. 2011. "Performance evaluation of seasonde highfrequency radar for vessel detection," Marine Technology Society Journal, vol. 45, no. 3, pp. 14-24.
G. Margarit, J. A. B. Milanes, A. Tabasco., 2009. "Operational ship monitoring system based on Synthetic Aperture Radar processing," Remote Sensing, vol. 1, no. 3, pp. 375-392.

S. Brusch, S. Lehner, T. Fritz, M. Soccorsi, A. Soloviev, and B.Van Schie., 2011. "Ship surveillance with TerraSAR-X," IEEE Transactions on Geoscience and Remote Sensing, vol. 49, no. 3, pp. 1092-1103,

H. Goldman and I. Bar-David., 1988. "Analysis and application of the excision CFAR detector," IEE Proceedings F - Radar and Signal Processing, vol. 135, no. 6, pp. 563-575.

S. I. Hwang and K. Ouchi., 2010. "On a novel approach using MLCC and CFAR for the improvement of ship detection by synthetic aperture radar, " IEEE Geoscience and Remote Sensing Letters,vol. 7, no. 2, pp. 391-395.

W. An, C. Xie, and X. Yuan., 2014. "An improved iterative censoring scheme for CFAR ship detection with SAR imagery," IEEE Transactions on Geoscience and Remote Sensing, vol. 52, no. 8, pp. 4585-4595.

G Gao, and G Shi., 2017. "CFAR Ship Detection in Nonhomogeneous Sea Clutter Using Polarimetric SAR Data Based on the Notch Filter," IEEE Transactions on Geoscience and Remote Sensing, vol. 55, no. 8,pp. 4811-4824.

G. Gao, L. Liu, L. Zhao, G. Shi, and G. Kuang., 2009. “An adaptive and fast CFAR algorithm based on automatic censoring for target detection in high-resolution SAR images," IEEE Transactions on Geoscience and Remote Sensing, vol. 47, no. 6, pp. 1685-1697.

K Ji, X Xing, H Zou, and J Sun., "A Novel Variable Index and Excision CFAR Based Ship Detection Method on SAR Imagery," Journal of Sensors, vol. 2015, pp. 1-10.

P. P. Gandhi and S. A. Kassam., 1988. "Analysis of CFAR processors in homogeneous background," IEEE Transactions on Aerospace and Electronic Systems, vol. 24, no. 4, pp. 427-445.

M. E. Smith and P. K. Varshney., 2000. "Intelligent CFAR processor based on data variability," IEEE Transactions on Aerospace and Electronic Systems, vol. 36, no. 3, pp. 837-847.

Khesali, E.; Enayati, H.; Modiri, M.; Mohseni Aref, M. Automatic Ship Detection in Single-Pol Sar Images Using Texture Features in Artificial Neural Networks. In Proceedings of the International Archives of the Photogrammetry, Remote Sensing and Spatial Information Sciences (ISPRS2015), Kish Island, Iran, 23-25 November 2015; pp. 395-399.

A. C. Frery, H.-J. M"uller, and S. J. S. Sant'Anna., 1997. “A model for extremely heterogeneous clutter," IEEE Transactions on Geoscience and Remote Sensing, vol. 35, no. 3,pp. 648-659.

A. Marino, M. Sugimoto, K. Ouchi, and I. Hajnsek. 2014 "Validating a notch filter for detection of targets at sea with ALOS-PALSAR data:Tokyo Bay," IEEE Journal of Selected Topics in Applied Earth Observations and Remote Sensing,vol. 7, no. 12 , pp. 4907-4918. 
The International Archives of the Photogrammetry, Remote Sensing and Spatial Information Sciences, Volume XLII-3, 2018 ISPRS TC III Mid-term Symposium "Developments, Technologies and Applications in Remote Sensing", 7-10 May, Beijing, China

R. Haralick, K. Shanmugam, and I. Dinstein., 1973. "Textural features for image classification,". IEEE Transactions on Systems, Man, and Cybernetics, vol.23, pp. 610-621.

M. Migliaccio, L. Mascolo, F. Nunziata, M. Sarti, and G. Mazzarella., 2014. "COSMO-SkyMed HH/VV pingpong mode SAR data to discriminate among sea, urban, and vegetated areas," IEEE Journal of Selected Topics in Applied Earth Observations and Remote Sensing, vol. 7, no. 7, pp. 2880-2894.

S. N. Anfinsen and T. Eltoft., 2011. "Application of the matrixvariate Mellin transform to analysis of polarimetric radar images," IEEE Transactions on Geoscience and Remote Sensing, vol. 49, no. 6 , pp. 2281-2295.

C. Zhu, H. Zhou, R. Wang., 2010. "A novel hierarchical methodof ship detection from spaceborne optical image based on shape and texture features," IEEE Transactions on Geoscience and Remote Sensing, vol. 48, no. 9, pp. 3446-3456.

L. Bruzzone, F. Roli, S. B. Serpico., 1995. "An extension of the Jeffreys-Matusita distance to multiclass cases for feature selection". IEEE Transactions on Geoscience and Remote Sensing, vol. 33, no. 6, pp. 1318-1321.

G. V. Camps and L. Bruzzone., 2005. "Kernel-based methods for hyperspectral image classification," IEEE Transactions on Geoscience and Remote Sensing, vol. 43, no. 6, pp. 1351-1362.

Y. Bazi and F. Melgani, 2006. "Toward an optimal SVM classification system for hyperspectral remote sensing images," IEEE Transactions on Geoscience and Remote Sensing, vol. 44, no. 11 , pp. 3374-3385. 